\title{
PHLPP1 phosphatase inhibition in hypothalamus restores insulin signaling and action and reduces adiposity in obese rats
}

\author{
Bruna dos Santos Cardoso*, Gisele Castro, Natália Ferreira Mendes, Paula Gabriele Fernandes Quaresma, \\ Tamires Marques Zanotto, Mario Jose Abdalla Saad, Patrícia de Oliveira Prada
}

From 20th Brazilian Diabetes Society Congress

Porto Alegre, Brazil. 11-18 November 2015

\section{Background}

Obesity results from imbalance between food intake and energy expenditure. The energy homeostasis is regulated by hypothalamic neurons that receive different neural, hormonal and metabolic signals. Insulin is one of the main hormones that regulate energy homeostasis and acts through a cascade of intracellular signaling that depends on the activation of several proteins, such as Akt. Our hypothesis is that $\mathrm{PH}$ domain and Leucine rich repeat Protein Phosphatase 1 (PHLPP1) inhibits Akt activity by dephosphorylating serine 473 residues, impairing the insulin action in the hypothalamus. However, PHLPP1 expression and the role played by it on the hypothalamus of diet induced obesity (DIO) animals are not fully understood.

\section{Objectives}

To investigate PHLPP1 protein expression in the hypothalamus of DIO rats and to assess whether the PHLPP1 silencing improves insulin action and decreases body adiposity.

\section{Methodology}

Eight-week-old male Wistar rats were distributed into two groups. One group was fed with standard diet and the other group with high-fat diet, during eight weeks. After this period, the hypothalamus of Chow and DIO animals were dissected for analysis of PHLPP1 protein expression. In another experiment, DIO animals were cannulated in the lateral ventricle and received intracerebroventricular (ICV) treatment to silence PHLPP1

\footnotetext{
* Correspondence: bs.cardoso@yahoo.com.br
} UNICAMP, São Paulo, Brazil 